\title{
Mathematical Models of Homogenization for a Rammed Earth Blend Made of Crushed Limestone and Cement in Linear Micro-poro-elasticity
}

\author{
Vittoria Strazzeri ${ }^{1, a}$, Ali Karrech ${ }^{2}$ and M. Elchalakani ${ }^{2}$ \\ ${ }^{1}$ University of Western Australia, School of Design, Perth WA 6009, Australia \\ ${ }^{2}$ University of Western Australia, School of Civil, Mining and Environmental Engineering, Perth WA 6009 Australia
}

\begin{abstract}
Rammed earth (RE) designates natural building materials that can contain stabilized or non-stabilized soils or crushed stone blends. RE materials are non-homogenous and their performance depends on their compositions, grain size distributions, porosity, and mode of preparation. This category of building materials has recently been subject of extensive studies, due to the increasing interest in sustainable construction, which have highlighted the potential and limits of its large uptake. Although it has been demonstrated that RE can be treated as multi-component materials [1] a full characterization based on micro-structural considerations is still lacking. Full understanding of the role of porosity in the overall material performance has not been addressed. However, extensive literature $[2,3,4,5]$ is available that can describe the overall mechanical behaviour of similar materials, based on the principle continuum micromechanics. This study applies the Mori-Tanaka homogenization scheme to characterize the linear elastic behaviour of cement-stabilized crushed-limestone-based material by treating it as a porous composite. The composite comprises a solid phase consisting of limestone grains bonded together with hydrated cement bridges and a fluid phases consisting of different families of pores. The microstructure of rammed earth samples has been observed using an optical microscope. The obtained images have been collected and processed to obtain the pore sizes and pore size distribution. Finally, these statistics along with the Mori-Tanaka homogenization scheme have been used to determine the overall elastic properties that depend on a damage parameter enclosing the pore sizes, density, and texture
\end{abstract}

\section{Introduction}

Rammed earth materials mainly contain stabilised and non-stabilised soils or crushed stone blends. They are multi-components, non-homogeneous and porous materials and their performance depends on their composition, grain size distribution, porosity, and mode of preparation [6]. RE building materials can be considered environment-friendly because they require little amounts of cement, generally ranging from 6 to $10 \%$ by mass. In the recent years there has been an increasing interest in the full understanding of the RE materials mechanics, because of the appeal of sustainable building technologies and RE has gained a renewed interest as a sustainable alternative to conventional building materials. An increasing number of research contributions have shown the advantageous properties of RE $[6,7]$ but its systematic characterisation using modern multi-scaling techniques is lacking [6]. In fact, the use of RE still relies on traditional mixing approaches without thoroughly optimising its overall properties based on micro-structural considerations.

It has been demonstrated that the loss of cohesion in failed earthen buildings mostly depends on the materials porosity [8]. A relevant approach to characterise earthen-based building materials has been applied by [1] who suggested that rammed earth may be treated as a non-homogeneous (or multi-components) materials, responding to the Reuss and Voigt models [1]. Therefore, it is reasonable that the principles of continuum micro-mechanics apply to characterise rammed earth materials.

The continuum micromechanics approach defines linear solutions to elastic problems that are originally non-linear. Considering a domain $\Omega$ embedding an inclusion i (or inhomogeneity), the displacement field is non-linear due to the presence of the inclusion. This implies that the macroscopic mechanical behaviour is related to the local (microscopic) mechanical behaviour [9]. This assumption introduces a scale transition problem; the study of the material behaviour at different scales gives different outcomes [10]. The scale transition problem is addressed by the theory of homogenisation [11], which states that the macroscopic behaviour of non-homogenous materials can be evaluated knowing the components' physical properties and the components arrangement (or material topology) [2]. A number of studies $[2,4,5,14]$ investigate the linear elastic

\footnotetext{
${ }^{\mathrm{a}}$ Corresponding author: vittoria.strazzeri@research.uwa.edu.au
} 
behaviour of various non-homogenous, multi-phase and polycrystalline materials by applying continuum micro-mechanics. A number of elastic problems [2, 12,17] have been solved by addressing the effects of different types of inclusions on the overall behaviour using the classic theoretical work of [13].

This study applies the continuum micromechanics approach to characterise a cement stabilised crushed limestone blend in linear elasticity. The Mori-Tanaka homogenisation scheme has been applied to determine an analytical definition of the elastic constants and the constitutive law of the material on the base of the analysis of the material's microstructure. RE is treated as a multi-phases material [11] comprising of a solid phase made of limestone grains and hydrated cement bridges and a fluid phase corresponding to the pore spaces. The full knowledge of the phase arrangement is essential to apply the above mentioned approach. The main objective is to define an overall elastic model that can describe the material's macro-mechanics by taking into account the role of porosity. The proposed model shows the variation of the homogenised stiffness tensor by varying the pore size and pore size distribution.

\section{The classic Eshelby problem for random media}

Eshelby [13] defined a solution to problem of infinite elastic domain $\Omega$ embedding an inclusion i. The solution provides a strain field, $\boldsymbol{\varepsilon}(\mathbf{x})$ when the infinite domain is subjected to a uniform boundary condition. The obtained strain field depends on the eigenstrain $\boldsymbol{\varepsilon}_{\mathbf{i}}$ that represents the deformation associated with the inclusion [12] as follows:

$$
\varepsilon(\mathbf{x})=S(\mathbf{x}): \varepsilon_{\mathbf{i}}
$$

where $\boldsymbol{S}$ is the position-related Eshelby's fourth order tensor. Eshelby found out that for an ellipsoidal inclusion, the strain field $\boldsymbol{\varepsilon}(\mathbf{x})$ becomes uniform in the domain $\Omega$ and the corresponding field $\boldsymbol{S}(\mathbf{x})$ becomes constant [12]. By refining the uniform eigenstrain field in the subdomain i, Eshelby's solution can be applied to solve problems in linear elasticity dealing with equivalent ellipsoidal inclusions, named as the Eshelby conjecture [12]. The linear solution to the classic Eshelby problem for random media represents one of the main findings of micromechanics since it relates the local strain to the macroscopic strain in the presence of the inclusion [11]. The solution has been adopted as a theoretical base to define a number homogenisation schemes, where it is instrumental to define the strain and stress concentration tensors relating the average local stress and strain tensors to the macroscopic ones [11]. In the case of multi-phase media the Eshelby ellipsoidal inclusion corresponds to an ellipsoidal pore space [11].

\section{The Mori-Tanaka homogenisation scheme}

The The Mori-Tanaka homogenisation scheme provides a solution to the Eshelby problem for multi-phase materials, considering the pores as inclusions and evaluating the interaction between them. Let's consider a domain of the material, $\Omega$, in which the RVE overall behaviour depends on the local elastic fields. The domain $\Omega$ is composed of a solid phase occupying the domain $\Omega_{\mathrm{s}}$ and a fluid phase occupying the domain $\Omega_{\mathrm{f}}$. The following assumptions are taken into consideration:

$$
\begin{aligned}
& \sigma=0 \quad \text { in } \Omega_{\mathrm{f}} \\
& \varepsilon \neq 0 \quad \text { in } \Omega_{\mathrm{f}}
\end{aligned}
$$

the stiffness tensors $\mathrm{C}$ and the compliance tensors $\mathrm{S}$ in the solid and fluid domains are respectively:

$$
\begin{aligned}
& \boldsymbol{C}_{f} \ll \boldsymbol{C}_{s} \quad \text { (c) } \\
& \boldsymbol{S}_{f} \gg \boldsymbol{S}_{s} \quad \text { with } \boldsymbol{S}_{s}=\left[\boldsymbol{C}_{s}\right]^{-1}
\end{aligned}
$$

The stiffness and compliance tensors are inverses of each other beyond the size of a representative volume element. The Mori-Tanaka formulation of the Eshelby problem, replaces the continuum boundary condition in term of macroscopic strain $\mathbf{E}$ with an analogue average strain $\mathbf{E}_{0}$ that describes the overall deformation of the solid phase surrounding the pore due to the presence of the pore itself. Hence, the strain average in the pore domain reads:

$$
\overline{\boldsymbol{\varepsilon}_{\mathbf{f}}}=(\boldsymbol{I}+\boldsymbol{P}: \delta \boldsymbol{C})^{-1}: \mathbf{E}_{0}
$$

where $\mathrm{I}$ is the fourth order unit tensor, $\mathrm{P}$ is the fourth order Hill tensor, $\delta \boldsymbol{C}=-\boldsymbol{C}_{\mathrm{S}}$ and $\boldsymbol{P}: \delta \boldsymbol{C}=-\boldsymbol{S}$ with $\boldsymbol{S}$ denoting the Eshelby tensor and $\mathbf{E}_{0}$ denoting the average strain in the solid phase around the pore, due to the presence of the pore. Therefore, Eq. (2) becomes:

$$
\varepsilon_{\mathbf{f}}=(\boldsymbol{I}-\boldsymbol{S})^{-1}: \mathbf{E}_{0}
$$

According to Eshelby, the average strain in the solid phase is equal to:

$$
\overline{\varepsilon_{\mathbf{s}}}=\mathbf{E}_{0}
$$

In addition, the average strain in the fluid and solid domains must meet the strain average condition $\overline{\boldsymbol{\varepsilon}}=\mathbf{E}$, which means that

$$
\varphi_{0} \overline{\varepsilon_{f}}+\left(1-\varphi_{0}\right) \overline{\varepsilon_{s}}=\mathbf{E}
$$

where $\varphi_{0}$ corresponds to the volume fraction of the fluid phase and the complementary quantity $\left(1-\varphi_{0}\right)$ is the volume of the solid phase. Combining Eq. (2), (3), and (5) yields the following relation between the macroscopic strain $\mathrm{E}$ and the strain average in the solid phase due to the presence of the inclusion $\mathbf{E}_{0}$ :

$$
\mathbf{E}_{0}=\left[\left(1-\varphi_{0}\right) \boldsymbol{I}+\varphi_{0}(\boldsymbol{I}-\boldsymbol{S})^{-1}\right]^{-1}: \mathbf{E}
$$


When several pores are taken into account, equation (5) reads:

$$
\mathbf{E}_{0}=\left[\left(1-\varphi_{0}\right) \boldsymbol{I}+\sum_{i} \varphi_{\mathrm{i}}(\boldsymbol{I}-\boldsymbol{S})^{-1}\right]^{-1}: \mathbf{E}
$$

where $\varphi_{\mathrm{i}}$ denotes the volume fraction of the inclusions $\mathrm{i}$. Replacing $\left(1-\varphi_{0}\right)$ with the solid volume $\varphi_{\mathrm{s}}$ equation (7) reads:

$$
\mathbf{E}_{0}=\left[\varphi_{\mathrm{s}} \boldsymbol{I}+(\boldsymbol{I}-\boldsymbol{S})^{-1}\right]^{-1}
$$

The stress can be expressed as $\boldsymbol{\sigma}(\mathbf{x})=\boldsymbol{C}_{s}: \boldsymbol{\varepsilon}_{\mathrm{s}}(\mathbf{x})$ within the solid domain and as $\boldsymbol{\sigma}(\mathbf{x})=\boldsymbol{C}_{i}: \boldsymbol{\varepsilon}_{\mathrm{i}}(\mathbf{x})$ within the inclusions [1]. The overall average reads:

$$
\begin{aligned}
& \boldsymbol{\Sigma}=\frac{1}{|\Omega|} \int_{\Omega} \chi_{\mathrm{s}} \boldsymbol{C}_{s}: \boldsymbol{\varepsilon}_{\mathrm{s}}(\mathbf{x}) d \Omega+\sum_{i} \int_{\Omega} \chi_{\mathrm{i}} \boldsymbol{C}_{i}: \boldsymbol{\varepsilon}_{\mathrm{i}}(\mathbf{x}) d \Omega= \\
& \varphi_{\mathrm{s}} \boldsymbol{C}_{s}: \mathbf{E}_{0}
\end{aligned}
$$

where $\chi_{s}$ and $\chi_{i}$ denote the characteristic functions of the phases. Therefore, the constitutive law for the overall domain reads:

$$
\begin{aligned}
& \boldsymbol{\Sigma}=\boldsymbol{C}_{\mathrm{hom}}: \mathbf{E}=\varphi_{\mathrm{s}} \boldsymbol{C}_{s}: \overline{\boldsymbol{A}_{s}}: \mathbf{E}=\varphi_{\mathrm{s}} \boldsymbol{C}_{s}:\left(\varphi_{\mathrm{s}} \boldsymbol{I}+\right. \\
& \left.+\sum_{\mathrm{i}} \varphi_{\mathrm{i}}\left(\boldsymbol{I}-\boldsymbol{S}_{\mathrm{i}}\right)^{-1}\right)^{-1}: \mathbf{E}
\end{aligned}
$$

where $\overline{\boldsymbol{A}_{s}}$ is the fourth order strain localisation tensor. Combining Eq. (9) with Eq. (10) the Mori-Tanaka estimation of the homogenised stiffness tensor reads:

$$
\boldsymbol{C}_{M T}=\varphi_{\mathrm{s}} \boldsymbol{C}_{S}:\left(\varphi_{\mathrm{s}} \boldsymbol{I}+\sum_{\mathrm{i}} \varphi_{\mathrm{i}}\left(\boldsymbol{I}-\boldsymbol{S}_{\mathrm{i}}\right)^{-1}\right)^{-1}
$$

\section{Cement stabilized crushed limestone blend}

The blend object of this study contains crushed limestone collected from the surrounding area of Perth (Western Australia) and general purpose cement obtained from Swan Cement and used for stabilisation. The raw material has been oven-dried to remove moisture, sieved to obtain the particle size distribution (Figure 1) and mixed such that the composition is $8.1 \%$ cement, $9.1 \%$ water and $82.8 \%$ by mass. The specimens have been manufactured and tested according to the procedure illustrated in the (Australian Standard, 2008). The results of the test indicate that the specimens (three of them have been tested and the average values are reported in this paper) reached a Young modulus of $646.49 \mathrm{MPa}$ and unconfined compressive strength (UCS) of $13.06 \mathrm{MPa}$ at 28 days of curing.

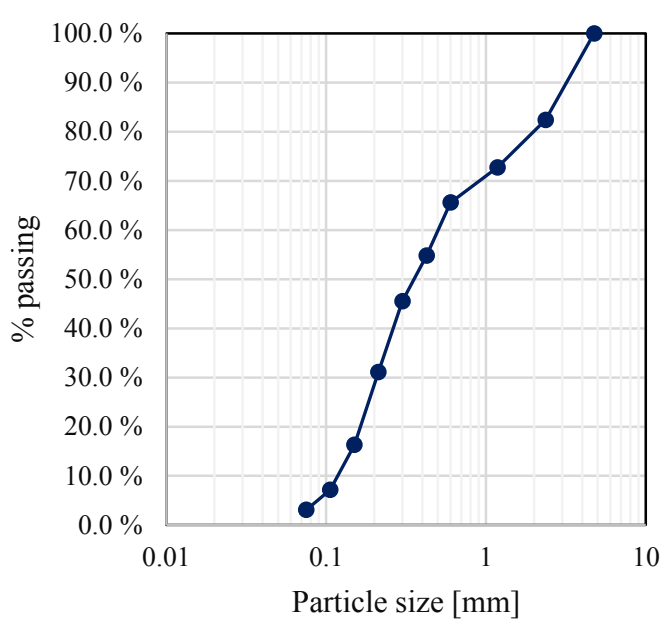

Figure 1. Particle size distribution.

\subsection{The material microstructure.}

The microstructure of the material has been observed and the pore size distribution has been evaluated. Fragments of crushed samples have been prepared for microscope observation. The fragments have been embedded in epoxidic resin, hardened, and polished to prepare them for subsequent observation. Two samples have been observed using a Leica DME optical microscope at a total resolution of $250 \mathrm{x}$; in total 30 pictures have been collected. The pictures have been processed using in-house python scripts to evaluate the pore size in terms of areas. The main image processing steps are de-noising the image by applying a median filter, applying digital masks to identify the pores, removing the non-representative voids and using mathematical morphology to estimate the pore dimensions. The output of the image processing of each picture is a vector containing the surface of the pores shown in the picture. Figure 2 shows an example of the image processing steps for one of the 30 pictures.
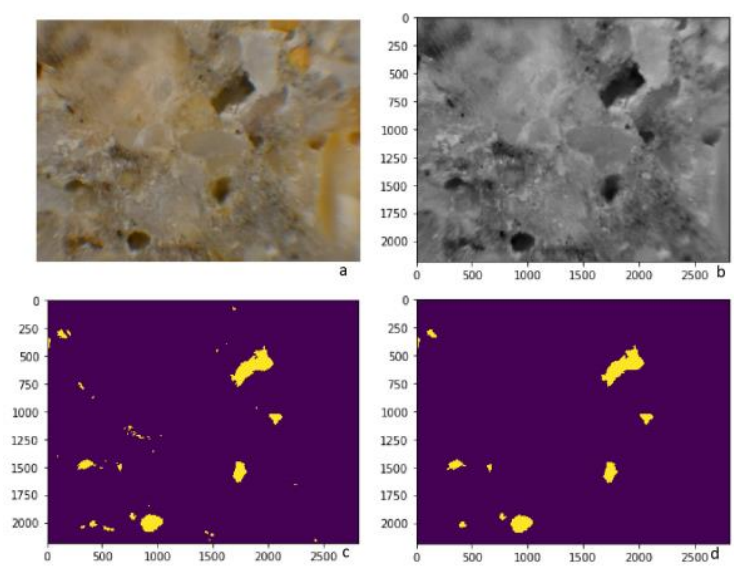

Figure 2. Steps of image processing.

In total, 30 vectors have been concatenated in a single vector containing the representative pore areas. The 
results in terms of pore areas, obtained using image processing have been subjected to statistical analysis to determine the pore size distribution. Figure 3 illustrates the workflow and indicates the correlation between the image processing and the statistical analysis.

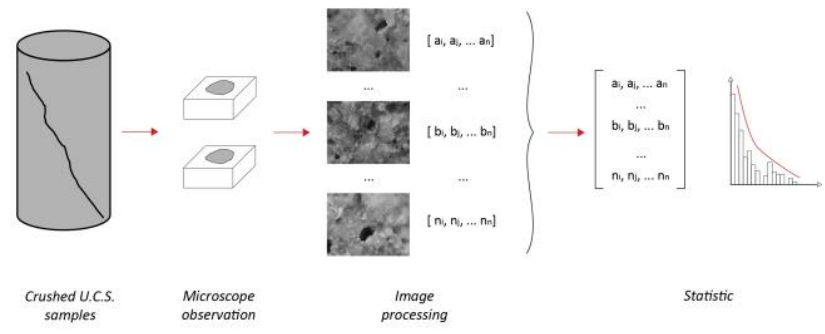

Figure 3. Workflow from UCS tests to statistic analysis.

\subsection{Pore size distribution.}

This study assumes that the domain $\Omega$ corresponds to a representative volume element of the material and that pores of different sizes are distributed randomly. The pore size distribution has been described by a continuous log-normal probability function that is defined on a positive support (defined from zero to infinite). This probability density function implies that the logarithm of the random variable-pore size-is normally distributed. Due to the central limit theorem, log-normal distribution allow to represent large numbers of randomly generated observations. Porosity in non-homogenous materials has already been assessed closed to a log-normal distribution [3]. The log-normally distributed random variable is defined as follow:

$$
X=e^{\mu+\sigma Z}
$$

where $\mu$ and $\sigma$ are the descriptive parameters of the distribution, being the mean and the standard variation respectively, while $Z$ is the standard normal deviate. $\mu$ and $\sigma$ are respectively location and scale parameters of the logarithm of the variable. The probability distribution function is given by

$$
f_{X}(x)=\frac{1}{x \sigma \sqrt{2 \pi}} e^{\left(-\frac{\ln x-\mu}{2 \sigma^{2}}\right)^{2}}
$$

Values for $\mu$ and $\sigma$ have been obtained by using a "fitdat "function on Matlab. Figure 4 illustrates the data histogram, Figure 5 shows the probability distribution function and Figure 6 shows the log-normal fitting for the dataset referring to pores areas. The moment of log-normal distribution are defined as

$$
E\left[X^{n}\right]=x^{n} f_{X}(x)=e^{n \mu+n^{2} \frac{\sigma^{2}}{2}}
$$

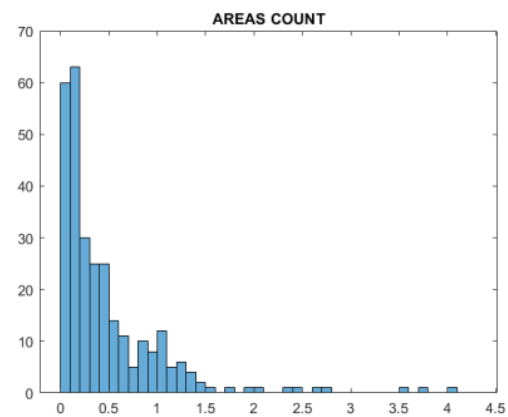

Figure 4. Data histogram.

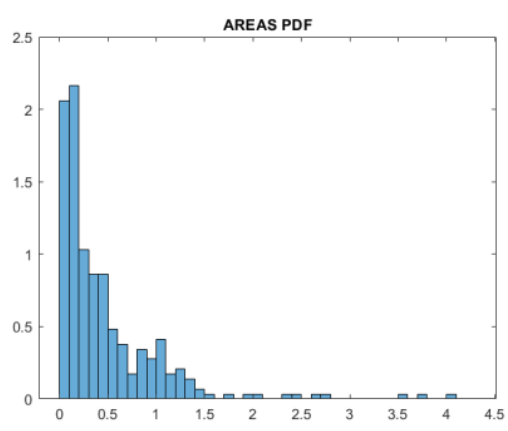

Figure 4. Probability distribution

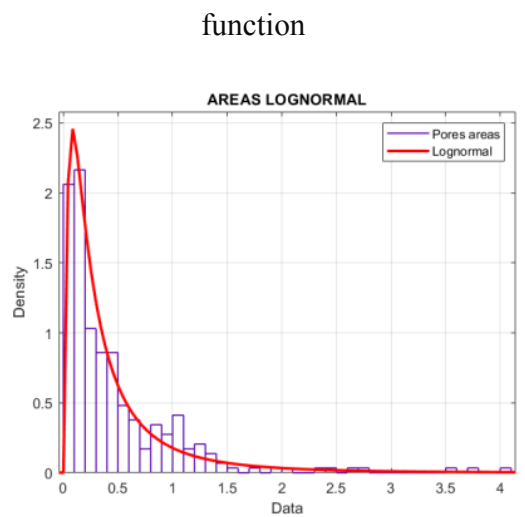

Figure 5. Lognormal fitting.

The representative porosity of the material object of the study has been evaluated by assuming that the pores are penny shaped, such that the volume of a given pore of size $\mathrm{x}$ is $\frac{4 \pi}{3} r_{\varepsilon} x^{3}$, where $r_{\varepsilon}$ is the ratio between the smallest dimension and the radius of the pore's flat surface $[4,5]$. Hence, if the pores are oriented randomly, the crack density $\left(f_{c r}\right)$ (number of pores per unit volume) can be expressed as follows:

$$
\int_{0}^{\infty} \frac{4 \pi}{3}\left(r_{\varepsilon} x^{3} f_{X}(x)\right) d x=f_{c r}
$$

\section{Estimation of the material's stiffness tensor}

In [4] the Mori-Tanaka homogenisation scheme has been applied to investigate non-linear behaviour of geo-materials. Since in the case of this study, the number of pores is large, we replace the discrete $\operatorname{sum} \sum_{\mathrm{i}} \varphi_{\mathrm{i}}(I+$ 
$\left.-S_{\mathrm{i}}\right)^{-1}$ by an integral to reflect the continuous nature of pore sizes. Eq. (11) can be rewritten as:

$$
\begin{aligned}
& \boldsymbol{C}_{M T}=\left(1-\varphi_{0}\right) \boldsymbol{C}_{S}:\left(\left(1-\varphi_{0}\right) \boldsymbol{I}+\int_{0}^{\infty} r_{\varepsilon}(\boldsymbol{I}+\right. \\
& \left.\left.-\boldsymbol{S}\left(r_{\varepsilon}\right)\right)^{-1} \frac{4 \pi}{3}\left(x^{3} f_{X}(x)\right) d x\right)^{-1}
\end{aligned}
$$

where the term $r_{\varepsilon}\left(\boldsymbol{I}-\boldsymbol{S}\left(r_{\varepsilon}\right)\right)^{-1}$ tends to finite tensor $\boldsymbol{T}$ as the ratio $r_{\varepsilon}$ tends to zero; the resulting fourth order tensor $\boldsymbol{T}$ is

$$
\boldsymbol{T}=\frac{1}{3}\left(Q_{1}-Q_{2}\right) 1 \otimes 1+Q_{2} \mathbb{I}
$$

where the constants $Q_{1}=\frac{16}{9} \frac{1-v^{s^{2}}}{1-2 v^{s}}$ and $Q_{2}=$ $\frac{32}{45} \frac{\left(1-v^{\mathrm{s}}\right)\left(5-v^{\mathrm{s}}\right)}{\left(2-v^{\mathrm{s}}\right)}$ depends solely on Poisson's ratio of the intact matrix $v^{\mathrm{s}}$. Since $\boldsymbol{T}$ is independent of the pore size, we obtain the overall fourth order elasticity modulus:

$$
\boldsymbol{C}_{M T}=\left(\frac{\kappa_{S}}{1+d_{\varepsilon} Q_{1}}-\frac{2}{3} \frac{\mu_{S}}{1+d_{\varepsilon} Q_{2}}\right) 1 \otimes 1+\frac{\mu_{s}}{1+d_{\varepsilon} Q_{2}} \mathbb{I}
$$

where $d_{\varepsilon}=\frac{4 \pi}{3\left(1-\varphi_{0}\right)} \int_{0}^{\infty}\left(x^{3} f_{X}(x)\right) d x$ is the damage parameter [4]. In the particular case where $r_{\varepsilon}$ is also independent of pore size Eq. (13) shows that $d_{\varepsilon}=\frac{r_{\varepsilon} \varphi_{0}}{\left(1-\varphi_{0}\right)}$. This assumption is not necessary to obtain Eq. (15), but it can be useful when the pore are regular. This is instrumental to measure an expression of $r_{\varepsilon}$ with respect to the pore size. The solution provides a strain field $\varepsilon(\mathrm{x})$, when the infinite domain is subjected to a uniform boundary.

\section{Conclusion}

This paper focuses on the heterogeneous nature of RE, a construction material that has been attracting a renewed acceptance in modern architectures given its sustainable character. A mathematical formulation based on continuum micro-mechanics has been used to describe the effective elastic properties of rammed earth. The results show that the inter-granular porosity and the distribution of pore spaces are key factors that influence the overall material properties of RE. It has been shown that the overall behaviour is also dependent on the solid matrix elasticity constants; this result is confirmed by the experimental data obtained through the uniaxial loading of rammed earth specimens. Since the knowledge of the microstructure of the material is the base of the above-mentioned model, this study has introduced a method to evaluate the representative porosity of rammed earth. The relevance of the proposed method lays in the fact that it links the direct observation of the material's microstructure to the statistic evaluation of the pore size distribution via image processing.

\section{References}

1 A. Karrech, C. Schrank, R. Freij-Ayoub and Regenauer-Lieb, IJMS, 78,1 (2014)

2 A. Standards, AS- 5101-4, Australian Standard, (2008).

3 K. V. Horoshenkov and M. J. Swift, JASA 11, 2371 (2001)

4 A. Karrech, F. Abbassi, H. Basarir and M. Attar, Mech. Mater. 104,107 (2017)

5 L. Dormieux, D. Kondo and F. Ulm, Microporomechanics (2006)

6 Q. Piattoni, E. Quagliarini and S. Lenci. Constr. Bui Mater 25, 2067 (2011)

7 J. Sanahuja, L. Dormieux and G. Chanvillard, Cement Concrete R. 37, 1427 (2007)

8 L. Miccoli, U. Muller and P. Fontana, Constr. Bui Mater. 61, 339 (2014)

9 C. Augarde, C. Beckett, J. C. Smith and A. J. Corbin, E3S Web of Conferences (2016)

10 R. Langenbach, JSEE 5, 133 (2004)

11 A. Zaoui, J. Eng. Mech 128, 808 (2002)

12 J. Christoffersen, M. M. Mehrabadi and S. Nemat-Nasser, J. Appl. Mech. 48, 339 (1981)

13 F. J. Ulm, G. Constantinides and F. H. Heukamp, Mater. Struct. 37, 43 (2004)

14 G. Voyiadjis and B. Deliktas, Acta Mech. 213, 3-26 (2010)

15 W. Zou, Q. He, M. Huang and Q. Zheng, J. Mech. Phys. Solids 58, 346 (2010)

16 J. D. Eshelby, Eshelby, Proceedings of the Royal Society of London. 241, 376 (1957)

17 W. Zou, Q. He, M. Huang and Q. Zheng, J. Mech. Phys. Solids 58,. 346 (2010) 